\title{
QU'EST-CE QUE C'EST UNE VIE JUSTE ET UNE VIE MUTILÉE ? LA CRITIQUE À L’ÉTHIQUE DE LA PENSÉE CHEZ ADORNO
}

EDUARDO JOCHAMOWITZ

\begin{abstract}
What is a damaged life? What kind of theoretical and metaphysical problems are challenged by Adorno's moral philosophy? In this paper we argue that the reflections delivered by Adorno after the catastrophe of the Second World War must be read from the scope of a systematic use of reason present in modern enlightenment philosophy as well as in modern market society. We claim the traditional concepts of moral autonomy and ethical life (Sittlichkeit) are both guided by the exchange principle developed by bourgeois society. We focus in the systematic architecture of Hegel's social philosophy with a view to identify an antimony shared with bourgeois society: the boundless expansion of the market and rational knowledge. The role of the synthetic concept of infinity is discussed in an ideological sense, stressing labor's division consequences. Finally, we set forth a discussion about-the limits of Adorno's theoretical criticism, especially on his use of dialectical categories and the ways in which they can be integrated in the core of a Critical theory.
\end{abstract}

\section{Introduction}

Theodor W. Adorno est l'un des rares philosophes du xxème siècle qui se sont attelés à la tâche philosophique du problème de la vie juste (Richtiges Leben). En récupérant une exigence longuement oubliée par les philosophes - faire de la réflexion éthique un mode de vie guidé par la critique - Adorno s'adresse aux lecteurs contemporains non seulement comme un critique de la phénoménologie et de l'idéalisme allemand mais avant tout comme un critique d'une praxis per-

https://doi.org/10.14712/24646504.2021.6

(C) 2021 The Author. This is an open-access article distributed under the terms of the

Creative Commons Attribution License (http://creativecommons.org/licenses/by/4.0). 
pétuée par la rationalité traditionnelle. ${ }^{1}$ Toutefois, l'extension du problème auquel s'adresse une réflexion sur la vie juste et sa contrepartie, la vie mutilée, ne va pas de soi. Comme nous argumenterons par la suite, cette forme de vie mutilée nous rappelle les dimensions de l'assujettissement aux impératifs économiques et rationnels d'un usage de la raison qui demeure l'unique critère disponible pour organiser la vie sociale engendrée par le capitalisme tardif.

Au premier abord, on pourrait rapporter la thématisation d'une vie mutilée à l'expérience vitale de la guerre et de la violence répétée tout au long du xxème siècle. Il est en effet bien connu qu'Adorno fit l'expérience de l'exil et de la dévastation de son pays natal, constatant l'ingérence - guère explicable - de la violence dans la continuité de la vie privée. Pourtant, ni le témoignage ni la remémoration de la biographie personnelle ne nous semblent représenter une condition suffisante permettant d'encadrer les exigences d'une réflexion éthique. À l'égard de cette expérience de dépossession, d'une existence qui semble être clôturée sur elle-même - en laissant pour le philosophe la fonction de penser l'extension des lésions comme la seule marge de liberté -, nous pouvons trouver dans les écrits d'Adorno une reconstruction théorique de cet enfermement même.

Il est important de remarquer qu'Adorno anticipe l'objection de la validité de sa propre critique et du lieu d'énonciation de sa critique. D'une part, il ne s'agit pas d'un acte de résistance individuel, de rester à l'écart de la vie quotidienne et de faire de la propre existence une pâle image de l'existence droite, même s'il n'est pas certain que cette image puisse représenter la vie juste sans régresser en une forme « [d']idéologie au service du propre intérêt ». ${ }^{2}$ D’autre part, l'élaboration d'une théorie critique, comme nous le verrons plus loin, doit se démarquer des catégories et principes de la théorie traditionnelle et d'un usage de la raison qui retrouvera à chaque synthèse une identité faite à sa mesure, même si elle en a temporairement besoin pour son propre développement.

Le point de départ d'un traitement philosophique de l'expérience sociale et d'une critique éthique passe nécessairement, comme nous l'affirmerons dans ce qui suit, I) par une considération de la dimension éthique qui est sous-jacente à tout idéal de vie juste. Elle s'inscrit dans un questionnement de l'éthique de la pensée qui conditionne l'horizon de la question du bien. A cela s'ajoute que II) le problème de la vie juste mobilise un examen des présupposés théoriques et des effets pratiques qui ont lieu lorsque la philosophie ne se conçoit plus comme un savoir qui prend en compte la négativité de l'expérience sociale. Nous finirons par

1 Sur la fonction sociale de la théorie traditionnelle et ses modes de transmission, $c f$. Horkheimer, Max : Théorie traditionnelle et théorie critique, Gallimard, Paris 1974.

2 Adorno, Theodor W. : Minima moralia : réflexions sur la vie mutilée, Payot, Paris 1981, 6. 
discuter le lien qu'Adorno dénonce dans sa Dialectique Négative, entre l'épuisement de l'autoréflexion de la philosophie et l'apparition d'une forme de vie mutilée au sein de la société de marché.

\section{De l'impossibilité d'une vie juste : trois modèles d'infidélité}

Une première façon d'aborder notre problème consiste à s'interroger sur la provenance de l'idée d'une vie juste. À cet égard, nous pouvons distinguer au moins trois grands modèles de vie juste mobilisés par l'histoire de la philosophie. Nous empruntons la catégorie de modèle car elle renvoie à la mise en valeur d'un cas singulier ou de la singularité qui ne devrait pas être masquée en raison de sa détermination historique intrinsèque. Un premier modèle se trouve pleinement développé dans la figure paradigmatique de Socrate, pour qui l'examen permanent de la propre vie devient le prérequis de la praxis du philosophe. L'idéal de sagesse de l'antiquité grecque, si nous nous fions à Giorgio Colli, était étranger au désir du savoir uniquement théorétique et demandait, en revanche, une considération particulière du parcours individuel, de la propre vie. ${ }^{3}$ La marque distinctive de ce modèle de vie juste se trouverait dans le refus à séparer l'action de la réflexion. Or cette séparation supprime la possibilité de thématiser éthiquement l'expérience vitale. Comme Adorno le signale dans son cours de 1962-1963, «le concept de la vie juste ne doit être séparé de celui de sagesse ${ }^{4}$, tandis que celle-ci demande sa réalisation par la voie de l'expérience subjective et perfectible. En ce sens, il est plausible d'affirmer que les causes de la condamnation de Socrate ne furent pas ses idées mais le mode de vie représenté par elles et leur divergence avec les modes de vie traditionnels de la cité.

En deuxième lieu, le modèle par excellence de la modernité : l'Aufklärung. En tant qu'impératif dirigé vers l'individu rationnel - rappelons-nous du mot d'ordre Sapere aude ! -, il envisage également une réalisation individuelle d'un idéal de la raison. Nonobstant, l'autonomie de la raison n'est plus guidée par l'idéal de la sagesse - par l'unité entre praxis et réflexion - mais plutôt par la détermination unilatérale de la théorie en dépit de la pratique et de l'expérience individuelle. Quand il est question de l'autodétermination du sujet - sous la forme d'un devoir-être confronté avec l'expérience du monde - nous nous confrontons avec une exigence exprimée négativement par la réflexion : malgré les circonstances du monde et les

Cf. Colli, Giorgio : La sagesse grecque 1, Éditions de l'éclat, Paris 1990, pp. 36-41.

4 Adorno: Philosophische Terminologie. Zur Einleitung, Suhrkamp, Frankfurt am Main 1973, p. 132. 
formes de vie traditionnelles, la raison commande à agir selon ses propres règles. Effectivement, nous pouvons limiter avec Kant un idéal de vie juste qui développe un usage critique de la raison, dirigé, en premier lieu, à transformer l'individu et, seulement en deuxième lieu le tout social. Par ailleurs, le reproche d'une « incapacité de se servir de son entendement sans être dirigé par un autre ", d'un état dont "lui-même est responsable ${ }^{5}$ vise précisément à réveiller, en chaque individu, une universalité inhérente à la pensée et réalisable par tous.

Le troisième modèle, une sorte de réponse aux modèles antérieurs, est celui du matérialisme historique. Dans la 11eme thèse sur Feuerbach, nous pouvons constater que le paradigme d'une vie juste suppose une transformation du monde par la voie de la praxis en vue de l'incapacité que présente la théorie au moment d'aller au-delà de l'interprétation. La tâche d'une telle transformation nous confronte à un modèle de vie juste dans la mesure où la praxis révolutionnaire, capable de réaliser ce que la philosophie n'a pas su faire, a besoin de l'expérience concrète des contradictions à être surmontées. Nous pouvons constater que, malgré la différence radicale de ce modèle, Marx continue à considérer le philosophe comme le responsable capable de réaliser, dans la singularité de sa condition historique, une nouvelle forme d'universalité et d'autonomie : le Gattungswesen de l'homme. ${ }^{6}$ Même si Marx constate l'insuffisance des modèles précédents, d'où résulte la dénonciation de la philosophie comme étant incapable de transformer le monde, la connaissance du philosophe restera une condition pour qu'une praxis transformatrice puisse avoir lieu.

Comme nous pouvons maintenant le constater, ces trois modèles de vie juste partagent une prémisse fondamentale : la mise en cause de l'éthique de la pensée en fonction de chaque configuration sociale et historique. En revenant sur les formulations classiques d'une totalité organique du corps social, élaborées par

5 Kant, Immanuel : Qu'est-ce que les Lumières?, in : CEuvres philosophiques, Vol. II, Gallimard, Paris 1980, p. 209.

6 Cf. Trân-vàn-Toàn : " Note sur le concept de 'Gattungswesen' dans la pensée de Karl Marx ", in Revue Philosophique de Louvain, Nr. 4, 1971, p. 535 : « Pour Marx l’homme est Gattungswesen en tant qu'être social. Cependant il ne l'est pas naturellement, immédiatement. Il a à se réaliser comme Gattungswesen dans un processus historique. [...] en tant qu'être pensant, l'homme saisit des objets universels et devient universel lui-même ; en tant que travailleur libre, il associe toute la nature à sa vie en s'intégrant lui-même dans l'histoire du genre humain. Cette histoire, les hommes ont encore à la réaliser ensemble consciemment, et ce n'est qu'à cette condition qu'ils deviennent vraiment hommes ». 
Aristote $^{7}$ et $\mathrm{Hegel}^{8}$, Adorno ne cesserait pas de tirer les conséquences de l'ingérence ou du primat du tout sur l'existence individuelle. "Dans la société individualiste, écrit Adorno au début de Minima Moralia, l'universel ne se réalise pas seulement à travers l'interaction des individus, mais la société est essentiellement la substance des individus $»{ }^{9}$ Disons tout d'abord que les conditions matérielles et symboliques de toute collectivité ne sont pas uniquement les conditions données de son existence effective, mais sont également ses conditions d'action possible. La société encouragée par le capitalisme, quant à elle, est une totalité conditionnée par les individus qui la composent, sauf que ce conditionnement n'est pas guidé par une spontanéité ou une autonomie inaliénable, mais en fonction de la rationalisation des objectifs socialement partagés. Dans ce cadre, «la critique du monde administré », critique d'une rationalité obsédée par le perfectionnement de la gestion des moyens, " constitue la présupposition de l'éthique ». ${ }^{10}$

Si Adorno élabore une réflexion sur la vie mutilée ${ }^{11}$, au lieu d'une phénoménologie du sujet - articulée par un Je certain de son vécu ou de son autonomie -, c'est en raison de la condition historique d'un sujet qui ne se retrouve plus dans son expérience et qui reste aliéné de son propre agir. À ce propos Rahel Jaeggi précise que :

le scandaleux n'est pas simplement que l'individu ne puisse pas se soustraire à sa pression conformiste, mais qu'il soit privé de la possibilité de pouvoir se comprendre, se déterminer et s'individualiser en référence à une société correcte, et comme membre de cette dernière. ${ }^{12}$

7 Cf. Bernstein, Jay: Adorno. Disenchantment and Ethics, Cambridge University Press, Cambridge 2001, p. 41 : « Like Aristotle, Adorno presupposes that ethical thought is a reflexive articulation of ethical experience, which itself is structured through ethical practices. This assumes that the ethical possibilities open to an individual are delimited by the state of the ethical world this individual inhabits: wrong life (the state of ethical world) cannot be lived rightly ».

$8 C f$. Vandenberghe, Frédéric: Une histoire critique de la sociologie allemande : aliénation et réification. Horkheimer, Adorno, Marcuse, Habermas, La Découverte, Paris 1998, p. 57 : "La présente époque constitue en quelque sorte la vérité du système hégélien : le sujet et l'objet sont effectivement identiques, mais leur identité est une identité satanique. Le sujet est absorbé par l'objet et par là même liquidé, disparais dans le système comme un objet spatial dans un trou noir ».

9 Adorno: Minima moralia..., op. cit., p. 12.

10 Adorno: Probleme der Moralphilosophie, Surhkamp, Frankfurt am Main 1997, p. 261.

11 Cf. Spurk, Jan : Pour une théorie critique de la société, Paragon/Vs, Lyon 2006, p. 160 : «Minima Moralia ne présente pas des réflexions plus ou moins résignées 'sur' sa vie de chercheur et d'intellectuel dans la société du xxème siècle, une sorte de méditation sur sa vie. Adorno réfléchit à partir de sa vie, c'est-à-dire à partir de son existence individuelle et singulière au sein d'une société concrète, sur lui-même, sur les autres et sur la société et pour tenter de les dépasser ».

12 Jaeggi, Rahel : «Une critique des formes de vie est-elle possible? Le négativisme éthique d'Adorno dans Minima Moralia ", in Actuel Marx, Vol. 38, Nr. 2, Presses Universitaires de France, Paris 2005, p. 151. 
Une première conclusion s'impose : en tant que mode de vie déficitaire, le problème de la société de l'après-guerre n'est pas exclusivement analysable en fonction des asymétries et injustices parmi ses membres. Considéré dans son ensemble, le champ social dont Adorno tire l'existence d'une vie mutilée se définit plutôt par sa capacité d'étouffer la capacité de réflexion et, par conséquence, de transformation du tout social.

Subséquemment, une deuxième caractéristique sous-jacente aux modèles de vie juste doit être considérée : la possibilité d'une véritable autonomie individuelle. Rappelons-nous que face aux modes de vie traditionnels - tributaires de la normalisation de l'hétéronomie collective - l'unité entre la pratique et la théorie, en ses différents rapports, est la condition nécessaire de la réussite d'une vie juste. Certes, cette unité implique un échange constant entre le singulier et la totalité sociale à laquelle ce premier appartient. Pourtant, la vie juste n'est pas possible dans une société non-libre. De même, l'autodétermination du sujet devient impossible, comme le constate sans répit Minima moralia en analysant les vécus plus proches ${ }^{13}$, au point " [qu']il n'y a pas de vie juste dans la fausse ». ${ }^{14}$ Avec l'expression vie fausse, il nous semble qu'Adorno thématise la réification de la conscience, celle d'un sujet incapable d'aller au-delà de l'immédiateté de ses rapports de production et de consommation, lieu exclusif de la réalisation de son être. En d'autres termes, la vie est fausse parce que la réalité sociale qui la soutient est elle-même fausse ou réifiée, marquée par l'absence de qualités concrètes et reproduite par une organisation du travail également abstraite. ${ }^{15}$ Nous pouvons donc déjà affirmer que le degré d'autonomie que chacun peut atteindre est aussi déterminé par le champ de possibilités auquel la conscience individuelle est assujettie. ${ }^{16}$ Qu'aucun individu ne puisse résister à l'organisation d'un monde tourné vers le profit individuel - selon

13 Cf. Adorno : Minima moralia..., op. cit., $\$ 17$ : « La liberté s'est concentrée en négativité pure et ce qu'on appelait à la fin du siècle 'mourir en beauté' s'est limité au souhait d'abréger l'avilissement infini de l'existence ainsi que la douleur infinie de l'agonie, dans un monde où depuis longtemps il $\mathrm{y}$ a bien pire à craindre que la mort $»$.

14 Ibid., $\$ 18$, p. 36.

15 Cf. Adorno : Dialectique Négative, Payot, Paris 1992, p. 151 : « Pour se consoler, le penser s’imagine facilement posséder la pierre philosophale dans la réduction de la réification, dans la réduction du caractère de la marchandise. Mais la réification elle-même est la forme de la réflexion de la fausse objectivité : centrer la théorie sur elle qui est une figure de la conscience, tend acceptable la théorie critique pour un point de vue idéaliste, pour la conscience dominante et l'inconscient collectif ".

16 Sur le rôle de l'idéologie dans la théorie critique je renvoie à l'article de Alvarenga, Raphäl : « L'apport de Theodor W. Adorno à une critique de l'idéologie dans le capitalisme tardif ", in Les Carnets du Centre de Philosophie du Droit, Nr. 124, 2006. 
l'impératif d'adaptation à la concurrence et l'indifférence à la solidarité - ouvre les portes à l'émergence de ce qu'Adorno nomme la vie mutilée.

Nous insistons sur la dimension historique dans laquelle la vie juste devient inatteignable puisqu'elle tend vers ce que Habermas a plus tard appelé la colonisation du monde vécu ${ }^{17}$, c'est-à-dire l'intervention des impératifs hypothétiques au cœur de la réflexion éthique. En anticipant l'hypothèse de son assistant, Adorno a évalué l'époque de la post-guerre comme un scénario où le progrès matériel et technique a, d'ores et déjà, démenti les impératifs moraux de la modernité ${ }^{18}$; une situation où « l'architecte introverti de la pensée vit dans la lune que les techniciens extravertis sont en train de confisquer $»{ }^{19}$ Toutefois, nous n'avons nullement affaire ici à un criticisme moral de la société contemporaine. A l'inverse, l'insistance sur les conditions qui rendent impossible la vie juste s'associe chez Adorno à un questionnement de l'éthique à partir de l'expérience sociale de l'individu. La méthode qu'Adorno mobilise à ce propos est inhérente à l'objet critiqué, autrement dit c'est l'expérience sociale et individuelle qui interroge l'organisation sociale à l'œuvre. À cet égard, Roger Foster explique que la stratégie d'Adorno implique la présentation d'un

compelling universal claim from within individual experience, by allowing that claim to work in a way that is 'almost the reverse' of subsumption. Whereas subsumption would involve the extraction of a generalizable rule from individual experience, this alternative presentation must find a way of constructing this relation in a way that does not transform the particular into the instantiation of a rule. ${ }^{20}$

Cette démarche est manifestement contraire au bien connu adagio hégélien du primat de la totalité, or l'individualité peut faire valoir sa nature excentrique et négative. En conséquence, le critère pour un questionnement de l'éthique sera fourni par la praxis du monde administré, mais aussi de l'individualité administrée, de telle sorte que la critique devient réflexive à cette praxis en question. ${ }^{21}$

$17 C f$. Haber, Stéphane : "Le 'monde de la vie' comme catégorie critique aujourd'hui ", in Cahiers philosophiques, Nr. 132, 2013, pp. 58-74.

18 Adorno : Minima moralia..., op. cit., \$17 : «La giffe [die Signatur] de notre époque, c'est que personne, sans aucune exception, ne peut plus déterminer lui-même son existence en un sens relativement transparent comme celui qui, auparavant, était donné au terme d'une estimation des conditions du marché. Par principe, tous sont objets, y compris les plus puissants ».

19 Adorno : Dialectique..., op. cit., p. 11.

20 Foster, Roger: «Lingering with the particular : Minima Moralia's critical modernism ", in Telos, Nr. 155, 2011, p. 89.

21 Cf. Adorno : Prismes, Critique de la culture et société, Payot, Paris 1986, p. 12 : « La critique de la culture ne peut comprendre que la réification de la vie n'est pas due à trop de Raison [Aufklärung] 
[...] dans la phase présente de l'évolution historique, l'objectivité massive du mouvement en cours réside en ce qui n'est encore qu'une dissolution du sujet - sans que déjà s'en soit dégagé un sujet nouveau - l'expérience individuelle prend nécessairement appui sur le sujet ancien, condamné par l'histoire, qui est encore pour soi mais qui n'est plus en soi. Un tel sujet croit encore être assuré de son autonomie, mais le néant que les camps de concentration ont infligé aux sujets atteint maintenant la forme même de la subjectivité. 22

D'après ce bilan, il devient plus clair que la vie mutilée - la contrepartie de la vie juste - s'inscrit dans les limbes toujours en expansion d'une rationalité qui donne à l'autonomie individuelle une existence secondaire et se reproduit dans la logique qu'organisent les institutions sociales. Comme l'affirme Foster, l'instrumentalisation et l'assujettissement impliquent que, dans la perspective de la totalité sociale, chacun est « cognitively, ethically, or socially significant only insofar as they instantiate or embody a value that does not derive from the character of this particular as a particular, and which can be equally well instantiated in an indefinite series of other items $» .{ }^{23}$ Ainsi, la vie mutilée essaie d'éclairer le péril d'un processus historique qui pointe vers l'épuisement de la conscience du possible et, parallèlement, de l'incapacité individuelle à se détacher des exigences de la substance sociale, source d'un assujettissement anonyme ${ }^{24}$. En outre, si l'individu dans une société de marché reste bien un être social, l'image de soi préconisée par le bourgeois - un Je inconditionné, une conscience pour soi - s'enracine et se nourrit de la fidélité - devenue pour Adorno une forme d'infidélité ${ }^{25}$ - aux modèles de vie signalés ci-dessus.

Cependant, la spécificité de l'évaluation d'Adorno ne s'épuise pas dans la considération d'une impuissance individuelle, plutôt elle se caractérise par le fait de discerner, dans l'examen de la praxis sociale et économique, l'origine d'un usage de la raison qui reproduit une telle impuissance. En suivant Jaeggi, puisque nous

et que les mutilations que l'actuelle rationalité particulariste [partikularen Rationalität] fait subir à l'humanité sont les stigmates de l'irrationalité totale ".

22 Adorno: Minima moralia..., op. cit., dédicace, p. 10.

23 Foster : "Lingering with the particular, art. cit., p. 86.

24 Cf. Adorno : Dialectique..., op. cit., p. 191 : «Quelle que soit l'action que l'individu ou le groupe entreprennent contre la totalité dont ils font partie, cette action est contaminée par ce que la totalité a de mauvais, et celui qui ne fait rien ne l'est pas moins. À cet égard le péché original s'est sécularisé. Le sujet particulier moralement sûr de lui échoue et devient complice parce que, prisonnier de l'ordre, c'est à peine s'il peut faire quelque chose pour surmonter des conditions qui en appellent à la nature morale [die ans sittliche Ingenium appellieren] et réclamer leur transformation ».

25 Adorno : Minima moralia..., op. cit., dédicace, p. 11 : « La fidélité à son propre niveau de conscience et d'expérience a constamment la tentation de dégénérer en infidélité, en refusant de voir ce qui transcende l'individu et d'appeler par son nom ce qui en fait la véritable substance». 
sommes incapables de nommer ce qui est profondément erroné dans notre vie quotidienne, il nous faut, toujours à la recherche d'une forme d'universalité collective et à nouveaux frais, retourner à l'examen des conditions mêmes de production du savoir pratique. Autrement dit, si le constat de la dissolution du sujet et de son autonomie dans une identité sociale fonctionnelle résultent de la conjonction d'une praxis philosophique et d'une praxis sociale, une voie possible pour sortir de l'enfermement du sujet consisterait en la révision de l'enchevêtrement entre théorie et pratique sur laquelle se reproduit le phénomène de la vie mutilée.

\section{Identité et échange : I'antinomie du marché et des idées}

Comme nous argumenterons, la praxis gnoséologique mise en place par la philosophie sociale de Hegel, afin de confirmer le principe d'identité spéculative, sera conçue comme la prolongation d'une praxis issue de la rationalité bourgeoise. En suivant cette hypothèse, nous voudrions affirmer que la critique de la vie mutilée et de l'impossibilité de mener une vie juste est également, dans le cas d'Adorno, le point de départ d'une mise en cause de l'idéalisme de Hegel, qui peut finalement être interrogé dans sa fonction normalisatrice de la vie sociale. Parmi les différentes critiques adressées à Hegel, nous voudrions ici attirer l'attention sur la complicité signalée par Adorno entre la procédure systématique de l'idéalisme absolu et le principe d'échange de l'économie de marché développé par le capitalisme.

Le lien qu'Adorno décèle entre la démarche de l'idéalisme hégélien et l'organisation marchande de la société bourgeoise se trouve dans leur caractère systématique, qui ne vise à rien d'autre qu'à une homogénéité entre ses parts. Si la forme que la réflexion prend - du fait de l'exigence d'articuler un système - autorise uniquement la présence des contenus que la conscience fournit, cette dernière, pour sa part, « est soulagée de ce qui contredit le monde extérieur ». ${ }^{26}$ Comme procédure générale, la pensée de l'identité « dit ce à quoi se rattache quelque chose, de quoi il constitue un exemplaire ou un représentant, donc ce qu'il n'est pas lui-même ". ${ }^{27}$ Ce faisant, tout au long du travail d'intégration de la différence, en chaque synthèse de ce qui semble opposé intervient donc une "volonté d'identité ${ }^{28}$, le substrat d'une pulsion d'autoconservation dirigée par la raison. Par voie de conséquence, la ratio de l'idéalisme absolu est ce qui définit - à l'avance - tous les contenus que le système pourrait intégrer.

26 Adorno : Dialectique..., op. cit., p. 171.

27 Ibid., p. 122.

28 Ibid., p. 120. 
L’empire de cette " volonté d'identité " s'aperçoit nettement dans la portée qu'Hegel octroie au concept d'infinité, noyau du mouvement réfléchissant. Cette thèse pourrait être formulée comme ceci : l'infinité est positive à la démarche du système, c'est-à-dire intégrée par son architecture. De ce fait, le système se caractérise comme un pur processus, comme une totalité toujours en expansion. Le moment problématique de cette démarche apparait une fois que tout contenu ou expérience sont intégrés dans l'appareil catégorique du système. Le particulier - qui pouvait transformer la totalité donnée - est subséquemment réduit à la condition d'un moment inessentiel. ${ }^{29}$ L'absence de bornes, l'exigence d'aller toujours au-delà de la finitude de l'expérience - au prix de conserver uniquement ce qui était déjà fixé par la pensée - sont les traits qu’Adorno reconnaît au cour de la praxis idéaliste. Toutefois, l'intérêt qu'Adorno porte à l'architecture systématique de l'idéalisme hégélien est concentré dans l'exploitation de l'antinomie entre les prétentions de totalité et d'infinité. C'est cette conjonction impossible entre la puissance de la raison et l'ordre de la pensée qui constitue "l'antinomie de l'essence idéaliste ". Malgré l'exigence bien connue de rester face à la négativité de la chose même, la dialectique de Hegel

[...] imite une antinomie centrale de la société bourgeoise. Celle-ci aussi doit, pour se conserver elle-même, pour rester égale à elle-même, pour 'être', s'étendre constamment, aller plus loin, repousser les limites toujours plus loin, n'en respecter aucune, ne pas demeurer égale à elle-même. ${ }^{30}$

Contre cette logique de la pensée extrapolée à une organisation sociale au service du marché31, la Théorie Critique d'Adorno nous rappelle la non-équivalence de ce qui est assujetti à l'identité. Or le principe d'identité n'est pas exclusivement

29 Cf. Adorno : Minima moralia..., op. cit., Dédicace, pp. 12-13 : «Que, dans la préhistoire de l'Humanité, la tendance objective s'impose par-dessus de la tête des hommes et même grâce à l'anéantissement de l'individuel, sans qu'ait été jusqu'à présent réalisée historiquement la réconciliation entre l'universel et le particulier construite dans le Concept, voilà qui prend chez Hegel une allure caricaturale : encore une fois, il opte avec une souveraine froideur pour la liquidation du particulier. Nulle part n'est mis en doute chez lui le primat du tout. Plus le passage de l'individuation réfléchissante à la totalité glorifiée est problématique, dans la Logique hégélienne comme aussi dans l'histoire, plus la philosophie se fait justification des choses telles qu'elles sont [das Bestehende] et s'accroche au char de le tendance objective triomphante".

30 Adorno: Dialectique..., op. cit., p. 28.

31 Cf. Marx, Karl : Le Capital, I, Presses Universitaires de France, Paris 1993, p. 667 : « Accumuler pour accumuler, produire pour produire, c'est dans ces termes que l'économie classique formulait la vocation historique de l'époque bourgeoise. [...] Si pour l'économie classique le prolétaire n'est qu'une machine à produire de la survaleur, le capitaliste n'est également qu'une machine à transformer cette survaleur en surcapital. Elle prend sa fonction historique très au sérieux ». 
une prémisse spéculative, en ce qu'il relève aussi bien de la logique de la division sociale du travail. De son impact dépend la possibilité d'un autre ordre du possible, «si on ne retenait plus à personne aucune partie de son travail vivant, l'identité rationnelle serait atteinte et la société serait au-delà du penser identifiant ».32 Mais face au développement des forces productives, dont le capitalisme ne se limite pas à un rôle strictement matériel ou économique, l'éthos de la société et sa capacité d'autoréflexion ne sont pas restées intactes.

Jusqu'ici, nous avons considéré l'idéalisme hégélien comme la prolongation de la praxis bourgeoise. Maintenant, il nous faut considérer cette volonté d'identité dans sa capacité régulatrice à l'intérieur de la société de marché. Comme principe d'organisation de la vie productive, elle suppose une abstraction générale des besoins et des activités des femmes et des hommes. Or toute particularité doit être en mesure de s'effacer pour laisser sa place à un schéma dans lequel tout est échangeable par le biais du marché. De ce fait, nous remarquons que la fongibilité absolue du marché n'est autre chose que le phénomène concret du principe d'identité. Comme l'écrit Adorno :

Le principe d'échange, la réduction du travail humain au concept universel abstrait du temps de travail moyen, est originairement apparenté au principe d'identification. C'est dans l'échange que ce principe a son modèle social et l'échange n'existerait pas sans ce principe ; par l'échange, des êtres singuliers et des performances non-identiques deviennent commensurables, identiques. L'extension du principe fait du monde entier de l'identique, une totalité. ${ }^{33}$

Si la démarche théorique de l'idéalisme s'accorde à la volonté d'identité - amalgame de praxis et de théorie mentionnée ci-dessus -, le principe normatif de la société bourgeoise s'exprime dans le libre échange parmi des membres inégaux, qui acceptent sans doute ni crainte, des échanges inégaux. À ce sujet, Fréderic Vandenberghe souligne la perspective sociologique qui serait sous-jacente au principe d'identité : "le principe d'échange est structurellement homologue au principe d'identité. Il en est, pour ainsi dire, l'instanciation sociale ". ${ }^{34}$ Certes, la praxis de l'idéalisme vise à « une extériorisation [Entäußerung] intégrale ${ }^{35} \mathrm{de} \mathrm{sa}$

\footnotetext{
Adorno : Dialectique..., op. cit., p. 120.

33 Ibid., p. 119. Cf. également p. 143 : «L'universalité du sujet transcendantal est celle de la connexion fonctionnelle de la société, d'un tout qui se conjugue à partir des qualités et des spontanéités singulières, les limite en retour au moyen du principe niveleur de l'échange et virtuellement, en tant qu'impuissance dans leur dépendance du tout, les exclut ».

Adorno : Dialectique..., op. cit., p. 19.
} 
propre préconception de la totalité sociale, faisant de l'échange l'épiphénomène de son propre concept. De surcroît, le besoin d'affirmer une harmonie sous-jacente à l'échange - en tant qu'expression matérielle de l'Entäußerung de la conscience peut être compris comme une réponse symptomatique issue de l'autonomisation des rapports de production par le capital. ${ }^{36}$ Mais plus loin Vandenberghe ajoute :

Telle est la version adornienne de la relation base-superstructure : l'abstrait universel (la société, le système, la totalité, le principe d'échange lui-même) en tant qu'essence nouménale est l'infrastructure immédiatement inconnaissable qui détermine, de part et d'autre, les phénomènes particuliers tels qu'ils apparaissent concrètement à la surface. ${ }^{37}$

À la différence de Vandenberghe, nous ne pensons pas que cette homologie structurelle entre l'identité et l'échange se circonscrit exclusivement dans le cadre d'une théorie de la connaissance de la réification, qu'au-delà de celle-ci il n'y aurait rien d'autre à penser. La liaison entre la démarche identitaire mobilisée par Hegel et l'expansion sans mesure de la société de marché que nous venons d'exposer ne nous semble pas aboutir dans la constatation ni d'un enfermement absolu de la société dans le marché, ni d'un anéantissement de la capacité réflexive de la substance sociale. Au contraire, l'enfermement dans ses propres prémisses est l'antinomie résultant du dispositif théorique de l'idéalisme. Comme le signale Adorno, « le cercle de l'identification, qui en définitive n'identifie toujours que lui-même, fut tracé par le penser qui ne tolère rien à l'extérieur ; son emprisonnement est sa propre œuvre $» .^{38} \mathrm{Si}$ la Théorie Critique développée par Adorno ne fait pas partie de ce " penser emprisonné " c'est grâce à la réflexivité qu'elle soutient à l'égard d'elle-même.

Pour Adorno il s'agit de choisir entre une forme de réflexion fermée par la structure de la pensée - qui prend en compte l'exigence de la non-identité du concept et du réel -, ou une réflexion qui fait de la fermeture son propre principe, ce principe étant la forme de ses contenus. ${ }^{39}$ Cette disjonction nous amène, dans

36 Cf. Marx : Le capital, op. cit., p. 547 : «L'industrie moderne ne considère et ne traite jamais comme définitif le mode actuel d'un procédé. Sa base est donc révolutionnaire, tandis que celle de tous les modes de production antérieurs était essentiellement conservatrice. [...] cet antagonisme fait naitre la monstruosité d'une armée industrielle de réserve, tenue dans la misère afin d'être toujours disponible pour la demande capitaliste ; qu'il aboutit aux hécatombes périodiques de la classe ouvrière, à la dilapidation la plus effrénée des forces de travail et aux ravages de l'anarchie sociale, qui fait de chaque progrès économique une calamité sociale ».

37 Vandenberghe : Une histoire critique..., op. cit., p. 81.

38 Adorno: Dialectique..., op. cit., p. 138.

39 Cf. Ibid., p. 120 : «La dialectique négative est liée comme à son point de départ, aux catégories suprêmes de la philosophie de l'identité. Dans cette mesure, elle-même reste fausse, participant de 
le premier cas, à l'affirmation de la Théorie Critique et, dans le deuxième cas, aux antinomies de la réflexion. La « morale de la pensée » 40 incarnée par la philosophie de Hegel - à savoir la fidélité à la négativité de la conscience et à la vérité du savoir spéculatif - ressemble au geste du baron de Münchhausen se tirant lui-même par les cheveux pour sortir d'un marécage : cette pensée veut donner sa juste place à la différence, mais en conservant toujours l'autorité de pouvoir dire le dernier mot.

Pour finir, nous souhaitons attirer l'attention sur le fait qu'une réflexion sur la vie mutilée peut être comprise comme un questionnement des différents usages de la raison qui rendent impossibles l'exercice d'une vie juste. Nous avons cerné cette impossibilité en repérant l'absence de limites dont témoigne la volonté d'identité. Dans sa dimension réflexive, l'idéalisme prépare le terrain pour l'émergence d'une vie mutilée, lorsqu'il se révèle incapable d'accomplir ses propres exigences en vue de la perte systématique de sa capacité réflexive. En effet, quand les rapports sociaux sont tangibles uniquement par le biais de leur identité avec la totalité, toute forme de négativité capable de reformuler l'articulation de la vie sociale est subséquemment anéantie. Dans sa dimension matérielle, le principe général de l'échange se réalise dans l'absence générale d'un questionnement sur l'inégalité reproduite par le marché. Comme nous l'indiquions au début de ce texte, la possibilité d'une vie juste se trouve dans le questionnement des effets pratiques de la pensée. "La philosophie qui parut jadis dépassée, se maintient en vie parce que le moment de sa réalisation fut manqué $»{ }^{41} \mathrm{Ce}$ bilan, que signe le frontispice de la Dialectique Négative, n'est pas uniquement une constatation d'ordre historique. Il nous semble, avant tout, une exhortation à un retour de l'obligation morale inhérente à la pensée, c'est-à-dire de savoir ce qu'elle fait lorsqu'elle réfléchit sur soimême, sur son historicité et sur son monde qui est le sujet de son examen.

Eduardo Jochamowitz est doctorant en philosophie en cotutelles à l'Université catholique de Louvain et à l'Université Toulouse - Jean Jaurès. Sa recherche doctorale porte sur la philosophie de l'aliénation et la philosophie de l'histoire.

la logique de l'identité, elle-même ce contre quoi elle est pensée. Elle doit se rectifier dans sa progression critique qui affecte les concepts qu'elle traite pour la forme, comme s'ils étaient aussi pour elle encore les premiers ".

40 Cf. Adorno : Minima moralia..., op. cit., $\$ 46$, p. 101.

41 Adorno: Dialectique..., op. cit., p. 11. 